\title{
Programas de intervenção para atividade física nas escolas brasileiras: revisão com base no modelo RE-AIM
}

\author{
Assistance programs for physical activity in Brazilian schools: a \\ review based on the RE-AIM model
}

Jaqueline Aragoni da Silva (https://orcid.org/0000-0001-6344-9073) ${ }^{1}$

Eliane Cristina de Andrade Gonçalves (https://orcid.org/0000-0002-6091-9612) ${ }^{1}$

Diego Augusto Santos Silva (https://orcid.org/0000-0002-0489-7906) ${ }^{1}$

Kelly Samara Silva (https://orcid.org/0000-0002-7356-1680) ${ }^{1}$

${ }^{1}$ Centro de Desportos,

Universidade Federal

de Santa Catarina. R.

Professora Maria Flora

Pausewang, Trindade.

88036-800 Florianópolis

SC Brasil.

jaqui_aragoni@hotmail.com

\begin{abstract}
The analysis of physical activity (PA) assistance programs is of major importance to ensure progress in the area. The Reach, Efficacy, Adoption, Implementation and Maintenance (RE-AIM) tool has been broadly disseminated in the literature. This study set out to identify $P A$ assistance programs among Brazilian students and analyze them using the RE-AIM tool. The search was conducted in the Lilacs, SportDiscus, SciELO, Scopus, Web of Science, EBSCOhot, PsycINFO and PubMed databases. After thoroughly researching titles, abstracts and studies, 15 articles (seven PA assistance programs) were included. The programs were conducted in the past decade at public schools in the South and Southeast of Brazil. Multicomponent (education and teacher training) strategies were used with positive results in the practice of PA. With respect to the results of the RE-AIM tool, it was found that the most reported dimensions were "reach" (74\%), followed by "efficacy" (48\%), "adoption" (43\%), "implementation" (35\%) and "maintenance" (5\%). The indicators most frequently mentioned were those related to the internal validity of the program: demographic and behavioral information of the target population, method of identification of the target population, sample size, participation rate and number of organizational units involved.
\end{abstract}

Key words Physical activity, Health, Review, Programs, Adolescent
Resumo Analisar programas de intervenção para atividade física (AF) é de grande importância para auxiliar o avanço na área. A ferramenta RE-AIM vem sendo amplamente difundida na literatura. Neste sentido, o presente estudo objetivou identificar programas de intervenção para AF em escolares brasileiros e analisá-los por meio da ferramenta RE-AIM. A busca foi realizada nas bases Lilacs, SportDiscus, SciELO, Scopus, Web of Science, EBSCOhot, Psycinfo e PubMed. Após leitura de títulos, resumos e estudos na íntegra, foram inclusos 15 artigos (sete programas de intervenção para AF). Verificou-se que os programas foram realizados nos últimos dez anos, em escolas públicas, com a maior parte nas regiões Sul e Sudeste do Brasil. Foram utilizadas estratégias multicomponentes (educativas e treinamento de professores), com resultados positivos na prática de AF. Quanto às dimensões do RE-AIM, verificou-se que "alcance" foi a mais relatada (74\%), seguida de "efetividade/eficácia" (48\%), "adoção" (43\%), "implementação" (35\%) e "manutenção" (5\%). Os indicadores mais mencionados foram aqueles referentes à validade interna do programa: informação demográfica e comportamental da população alvo, método de identificação da população -alvo, tamanho da amostra, taxa de participação e número de unidades organizacionais envolvidas. Palavras-chave Atividade física, Saúde, Revisão, Programas, Adolescente 


\section{Introdução}

A prática regular de $\mathrm{AF}$ é recomendada desde a infância, principalmente como fator de promoção da saúde ${ }^{1}$. Nos primeiros ciclos da vida, o ambiente escolar é um local propício para estimular AF, uma vez que grande parte das crianças e dos adolescentes frequenta este ambiente ${ }^{2}$. De fato, intervenções internacionais ${ }^{3}$ e nacionais ${ }^{4,5}$ têm sido desenvolvidas nesse contexto. Porém, a maioria delas é aplicada sem antes identificar o perfil daqueles que serão beneficiados e a viabilidade da aplicação do programa para a realidade explicitada ${ }^{6}$.

Estudos de intervenção como "Academia da Cidade", em Recife (PE), "Academia da Cidade", em Aracaju (SE) $)^{7}$ e o "CuritibAtiva" têm sido avaliados metodologicamente por diferentes estratégias, a citar: aplicação de questionários padronizados, entrevistas estruturadas e semiestruturadas e inquéritos telefônicos. Apesar de estas avaliações serem iniciativas de programas importantes, apresentam limitações que impedem a identificação da validade interna e externa do estudo, como por exemplo, escassez de informação referente à relação causa-efeito da intervenção e falta de avaliação em longo prazo $^{6}$, o que pode dificultar a compreensão do tamanho do impacto dessas intervenções na saúde pública.

Dentre os diferentes processos avaliativos para estudos de intervenção, o modelo RE-AIM (em inglês: Reach, Efficacy, Adoption, Implementation and Maintanance) tem sido atualmente um dos modelos de avaliação mais requisitados para a área de $\mathrm{AF}$ e saúde 9 . Isso porque, o $\mathrm{RE}$ -AIM, desenvolvido nos Estados Unidos pelos pesquisadores Russel Glasgow, Thomas Vogt e Shawn Boles ${ }^{10}$, foi criado como proposta de modelo de avaliação de programas de promoção da saúde. O objetivo dessa proposta é auxiliar os pesquisadores e gestores no planejamento e na avaliação das ações, a fim de identificar elementos essenciais para construção de programas eficazes e efetivos ${ }^{11}$. Traduzido e adaptado para a realidade brasileira ${ }^{11}$, esse modelo é constituído por cinco dimensões (Alcance, Adoção, Implementação, Efetividade/Eficácia e Manutenção) que se relacionam entre si, e quando bem-sucedidas, potencializam o impacto das intervenções na saúde pública ${ }^{10}$.

Levantamento realizado com o objetivo de identificar estudos de intervenção para a promoção da saúde na escola verificou que, dos 32 estudos analisados, "eficácia/efetividade" foi o único componente do modelo RE-AIM relatado ${ }^{12}$. Esse fato demonstra que os estudos de intervenção com foco na promoção da saúde raramente relatam os itens do RE-AIM referentes à validade externa (taxa de participação e representatividade, por exemplo), o que limita a extrapolação dos resultados para uma possível aplicação prática pelos gestores ${ }^{12}$.

Revisão sistemática sobre intervenções em AF com adolescentes mostrou que dos 50 estudos incluídos, apenas 3,1\% tinham sido realizados em países de baixa ou média renda, sendo dois oriundos do Brasil ${ }^{3}$. Também são escassos os estudos que utilizaram o método RE-AIM como modo de avaliação de programas voltados à prática de AF, principalmente em adolescentes ${ }^{13}$. Ademais, foi encontrada na literatura somente uma revisão sistemática que analisou artigos nacionais sobre programas de AF com base no modelo RE-AIM, porém a população-alvo foram os idosos.

Com base no exposto, analisar os programas por meio do modelo avaliativo RE-AIM é de grande valia uma vez que a identificação do atendimento aos critérios estabelecidos pelo modelo permite potencializar o impacto desses programas ${ }^{6}$. Ainda, a utilização desta ferramenta aumenta o entendimento sobre os mecanismos que possibilitam a expansão dos programas para diferentes contextos e populações. Também é possível identificar se os programas responderam ao objetivo esperado, se foram realmente efetivos a ponto de haver manutenção das atividades estabelecidas e se houve efeito favorável na população investigada em longo prazo ${ }^{14}$. Tais investigações são extremamente necessárias nos dias atuais, posto que poderão ser informações relevantes do impacto das intervenções e dos critérios de validade?.

Dessa forma, propõe-se identificar os programas de intervenção para AF realizados em escolares de 10 a 19 anos e analisá-los por meio do modelo RE-AIM. Justifica-se o desenvolvimento deste trabalho baseado na necessidade de ampliar o escopo de conhecimento sobre intervenções que são efetivas e apropriadamente contextualizadas, principalmente em países de baixa e média renda $^{15}$. Uma das maneiras de contribuir para a expansão de implementação de intervenções é por meio da identificação do cenário atual do país, o qual pode ser obtido por meio de revisão sistemática ${ }^{9}$. Assim, revisar os programas para AF em escolas brasileiras proporcionará uma base de informações sobre o quanto pesquisadores ainda precisam avançar, em termos de implementação. Ainda, o atual estado da arte caracteriza-se como ponto de partida para futuras intervenções 
de base escolar, identificando quais informações ainda carecem nesta área de investigação.

\section{Método}

A revisão sistemática foi realizada em janeiro de 2016, usando oito bases eletrônicas: (PubMed, SciELO, Lilacs, Scopus, Web of Science, EBSCOhost, PsycINFO e SPORTDiscus). A busca foi realizada com os seguintes descritores em Ciências da Saúde (DeCS): "planos e programas de saúde"” or "estudos de intervenção" or "educação em saúde" or "intervenção" or "promoção da saúde". Para a busca referente a população do presente estudo (amostras cuja média de idade dos indivíduos estivesse entre 10 a 19 anos), os descritores utilizados foram: "adolescentes" or "jovens" or "estudantes" or "escolares" or "adolescência" or "alunos". Em relação a busca sobre intervenções direcionadas à AF, os descritores foram: "atividade física" or "exercício fisico" or "atividade motora". Além desses, foram utilizadas as palavras-chave: "escola" or "ambiente escolar" or "escolar" para certificar que os estudos eram realizados nas escolas brasileiras. Os mesmos unitermos também foram utilizados na língua inglesa, pois considera-se que alguns estudos, mesmo desenvolvidos no Brasil, sejam publicados em outra língua. Os descritores foram combinados por meio dos operadores And e Or. Não foram utilizados filtros para ano de publicação.

Foram adotados os seguintes critérios de inclusão: ser artigo original; intervenções realizadas em escolas do Brasil, com objetivo (primário ou secundário) de fomentar a prática de AF; amostras cuja média de idade dos indivíduos estivesse entre 10 a 19 anos. Foram excluídas as intervenções que envolvessem exclusivamente adolescentes obesos e/ou sobrepesos $(n=2)^{16,17}$.

Os artigos deste estudo foram selecionados por meio de método sistemático, em que um autor (JAS) realizou as seguintes etapas: Primeiramente, fez-se a exclusão dos artigos duplicados. Em seguida, foram lidos os títulos e resumos dos artigos. Na sequência, o texto completo dos artigos foi obtido, lido e incluído se estivessem de acordo com os critérios de inclusão. As referências de todos os artigos selecionados foram examinadas para identificar outras publicações que deveriam ser revisadas e incluídas. Quanto à seleção e avaliação das referências, estas foram realizadas por dois pesquisadores (JAS e ECAG) familiarizados com a metodologia. Caso houvesse divergência entre os dois pesquisadores, os artigos eram relidos e reavaliados para verificar qual seria a análise final. A Figura 1 traz o fluxograma do processo de busca, seleção e os respectivos motivos de exclusão das referências.

Para a checagem das publicações, foi utilizada a ferramenta RE-AIM, a qual permite capturar a forma com que os estudos de intervenções reportam questões relacionadas a fatores de validade interna e externa. Neste sentido, é possível verificar as cinco dimensões do modelo que são: "alcance": Número absoluto, proporção e representatividade dos indivíduos dispostos a participar do programa ou intervenção, comparados àqueles que desistem ou àqueles potencialmente elegíveis (características gerais da população da área); "eficácia/efetividade": Impacto da intervenção sobre os desfechos importantes (como nível de AF, qualidade de vida, mudança de comportamento), incluindo potenciais efeitos negativos; "adoção": Número absoluto, proporção e representatividade das organizações e dos agentes de intervenção dispostos a iniciar determinado programa ou intervenção; "implementação": em nível organizacional refere-se à fidelidade dos agentes de intervenção para os vários elementos do protocolo da intervenção, incluindo a consistência no fornecimento conforme previsto, o tempo e o custo da intervenção. Em nível individual refere-se à medida em que os participantes utilizam as estratégias da intervenção; "manutenção": Em nível organizacional refere-se à medida em que o programa ou intervenção torna-se institucionalizado ou parte das práticas de rotina e políticas organizacionais. Em nível individual refere-se à manutenção que tem sido definida como os efeitos benéficos em longo prazo (seis meses ou mais após o término da intervenção ${ }^{11}$.

Cada dimensão possui diferentes componentes, os quais foram analisados em cada estudo, obtendo um status de "sim" ou "não. A partir daí, verificou-se o percentual de estudos que atendeu a cada item, obtendo uma média para cada componente, bem como para a respectiva dimensão ${ }^{18}$.

\section{Resultados}

\section{Seleção dos artigos}

Foram localizados 1.287 artigos nas bases de dados, sendo que destes, 185 artigos foram excluídos pelo fato de serem duplicados. Dentre os 1.452 artigos que permaneceram 1.417 foram excluídos com base na leitura de títulos e resumos. Desta forma, foi realizada e leitura na íntegra de 


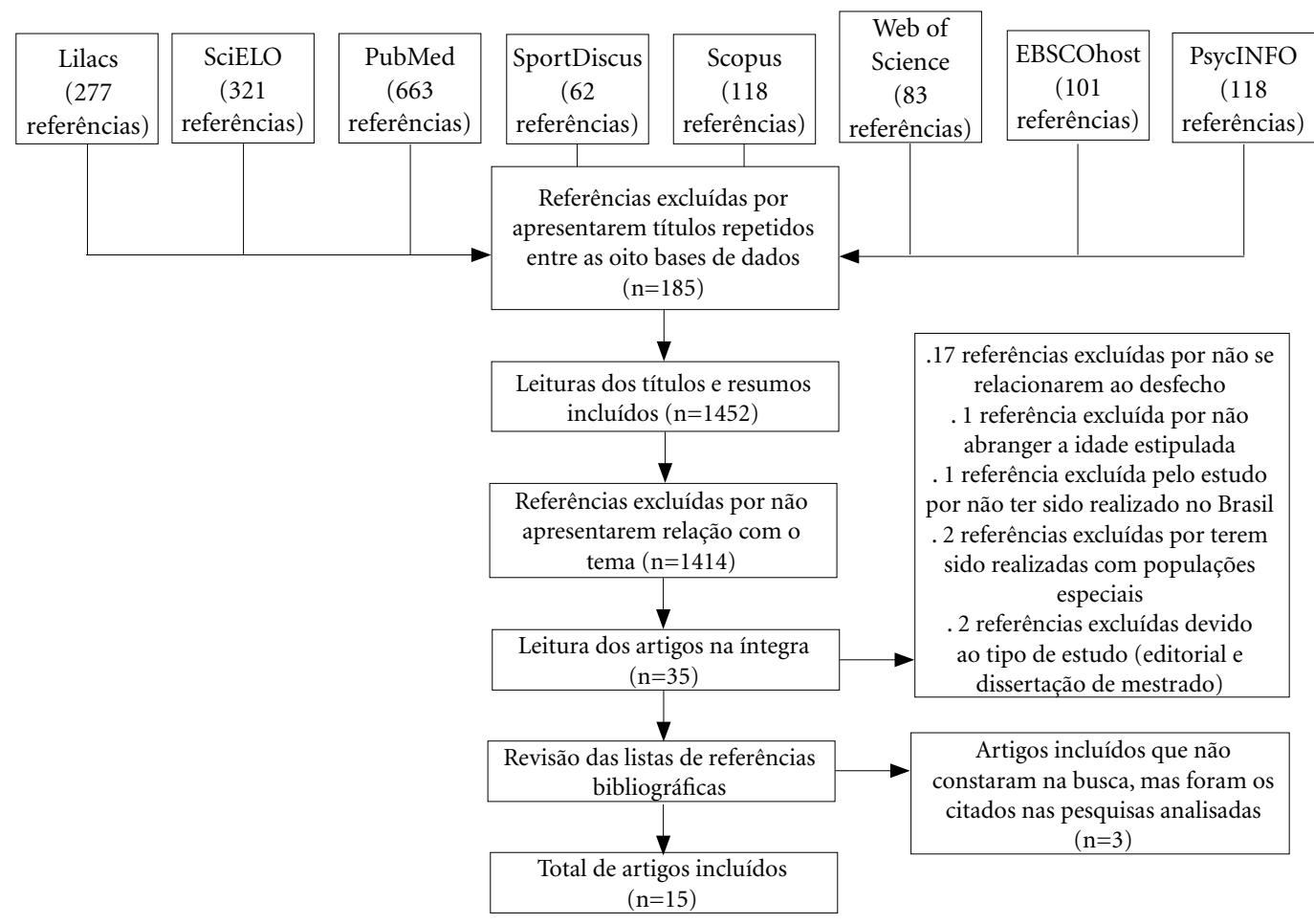

Figura 1. Busca, seleção e exclusão dos estudos sobre programas de intervenção direcionados a educação em saúde realizados nas escolas brasileiras com adolescentes de 10 a 19 anos.

35 artigos e, dentre os mesmos, 12 estudos atenderem aos critérios de inclusão. Por fim, após checar as referências destes, foram adicionados três artigos, totalizando 15 estudos inclusos nesta revisão.

Deste total, alguns estudos eram referentes aos mesmos programas de intervenção. Sendo assim, foram agrupados e analisados de forma conjunta, com o objetivo de complementar as informações das respectivas intervenções: "Saúde na Boa"4,19-23, "Hábitos Saudáveis, Meninas Saudáveis"24,25 e "Fortaleça sua saúde" ${ }^{\text {5,26 }}$ totalizando sete programas de intervenção para AF.

\section{Descrição dos Programas de Intervenção}

Quanto ao ano, os estudos aconteceram no período de 2006 a 2015, cuja maioria foi realizada nas regiões $\mathrm{Sul}^{27,28}$ e Sudeste do país ${ }^{24,25,29,30}$. Foi encontrado apenas um programa de intervenção na região nordeste $e^{5,26}$. O programa Saúde na Boa $^{4,20-23,31}$ englobou escolas tanto do Sul quanto do Nordeste do Brasil (Quadro 1).

Todos os estudos foram realizados em escolas públicas, sendo que destes, $u^{29}{ }^{29}$ incluiu também escolas particulares. A maioria dos programas incluiu nas amostras adolescentes ${ }^{5,24-26,28,30}$ ou crianças e adolescentes ${ }^{27,29}$. O tamanho amostral variou de 69 a 5.366 sujeitos (Tabela 1).

Os objetivos das intervenções foram, além do aumento da prática de AF, a promoção da saúde, ${ }^{5,26,28}$ hábitos alimentares, ${ }^{4,19-25,27,29,31}$ comportamento sedentário ${ }^{5,26,29}$ e prevenção da obesidade e excesso de peso ${ }^{24,25,27,30}$ (Quadro 1).

Todos os programas utilizaram grupos de controle e apenas um estudo relatou não utilizar amostra randomizada ${ }^{30}$. De maneira geral, os programas utilizaram estratégias com diversos componentes. As ações educativas foram citadas por todos os estudos e a realização de formação/ treinamento de professores foi uma ação utilizada pela maioria ${ }^{4,5,19-23,26,28,29,31}$ (Quadro 1).

Resultados positivos quanto ao comportamento relacionado à $\mathrm{AF}$ foram verificados na maior parte dos estudos ${ }^{4,5,19-26,29-31}$. Um estudo não identificou melhoria na prática de $\mathrm{AF}$, contudo, houve melhoria no conhecimento sobre este comportamento $^{28}$. Por fim, não estavam disponíveis os resultados de um programa de intervenção ${ }^{27}$ (Quadro 1). 


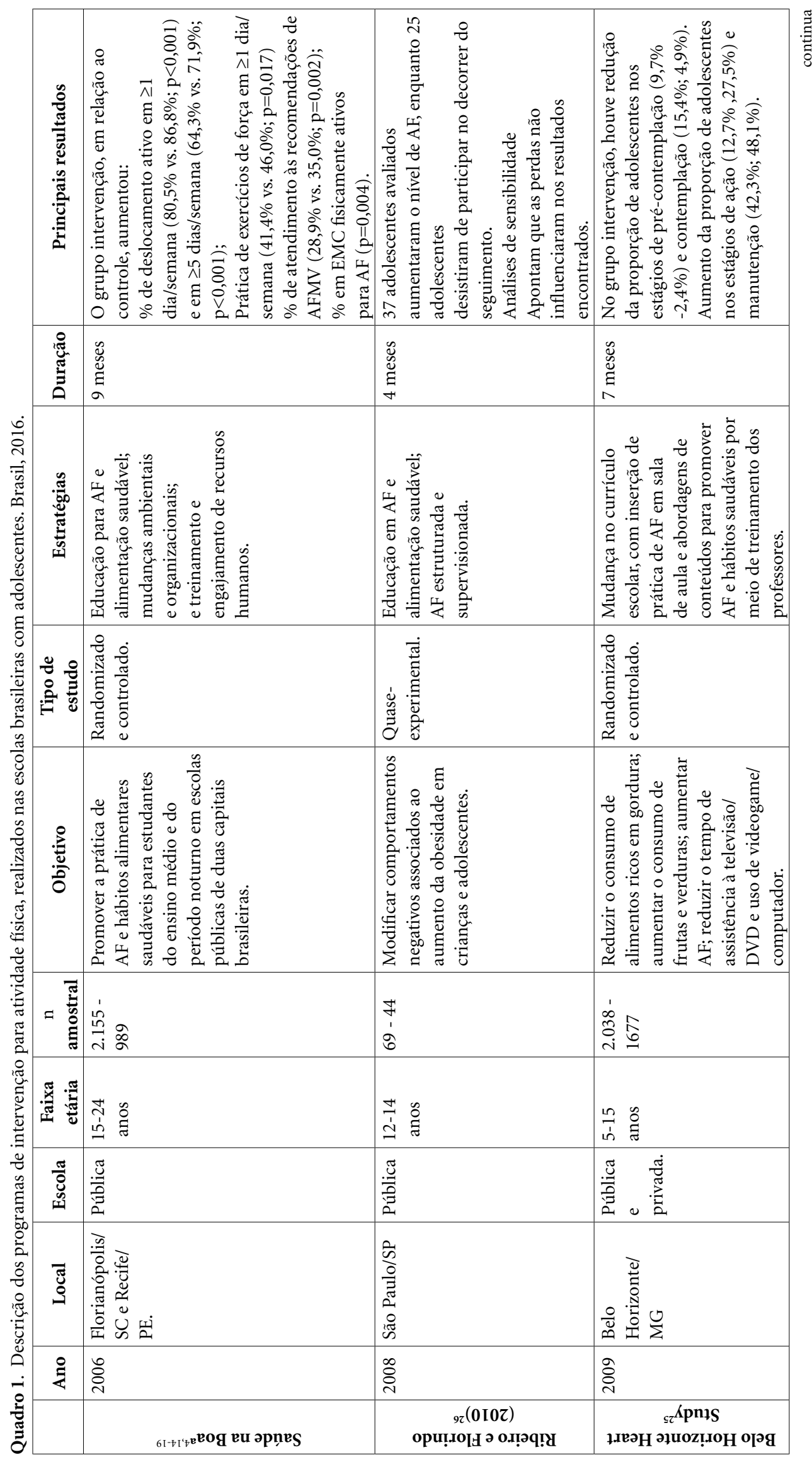




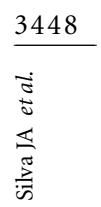

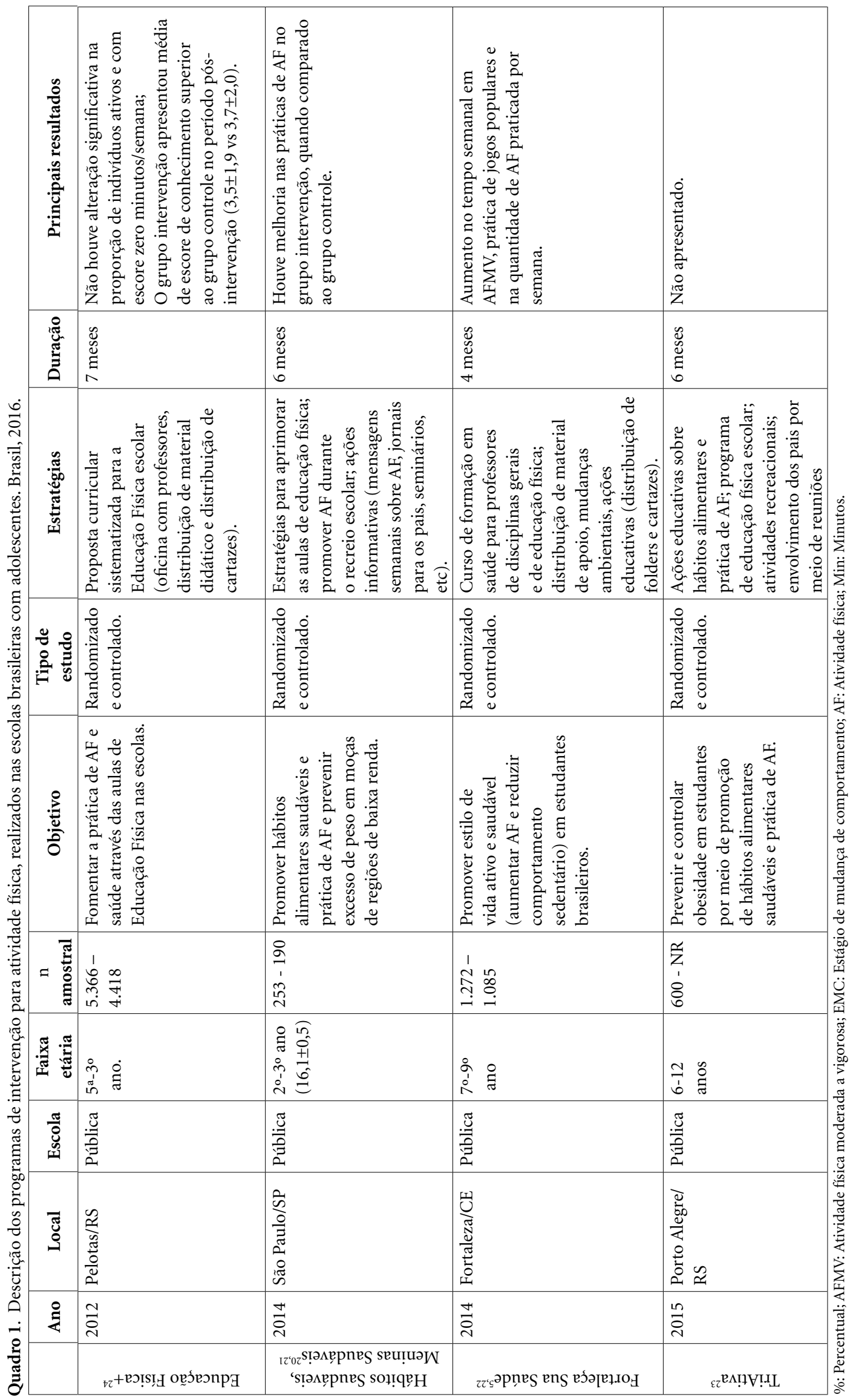




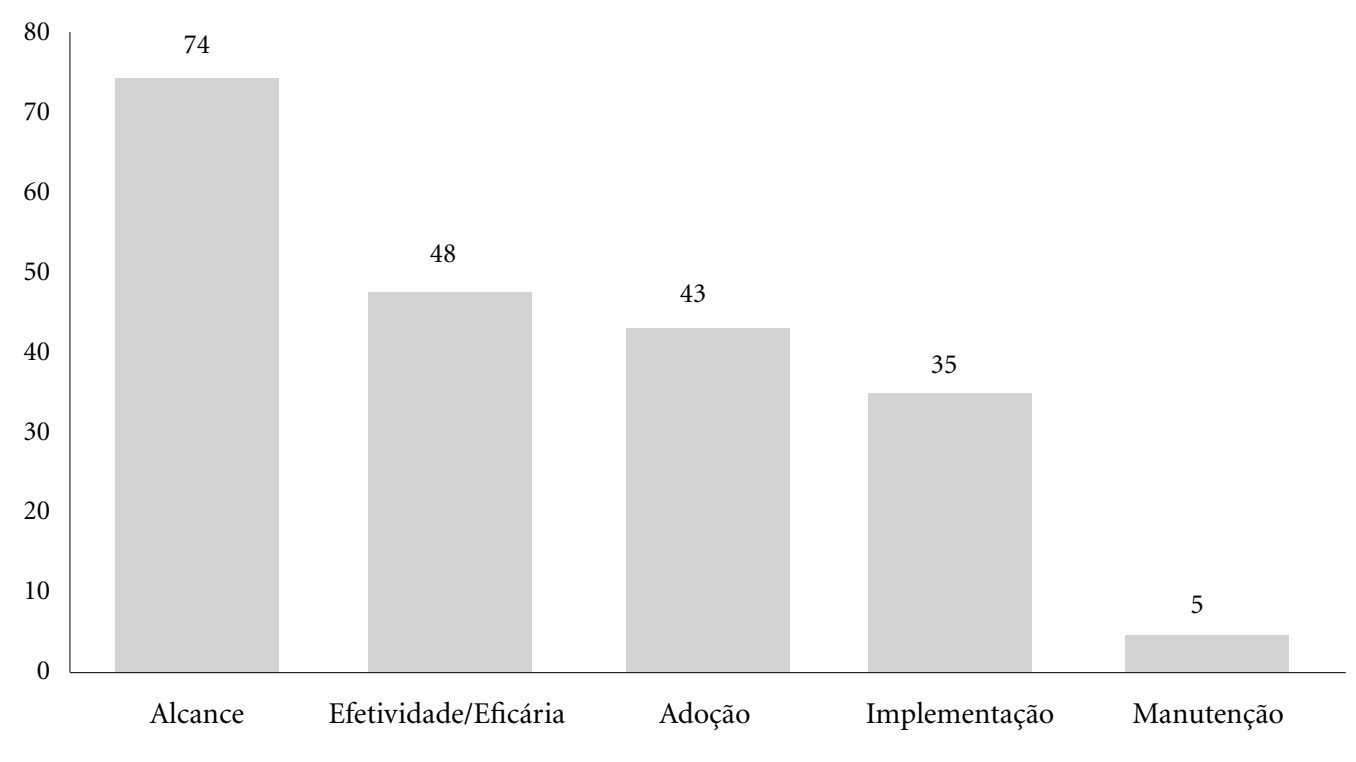

Figura 2. Proporção de detalhamento das dimensões do modelo RE-AIM pelos programas de intervenção para atividade física em escolas brasileiras.

\section{Descrição das dimensões do RE-AIM}

De acordo com a Figura 2 é possível verificar que, em média, 74\% dos indicadores de "alcance" foram descritos nas publicações. Na sequência estiveram os indicadores de "efetividade/eficácia" (48\%), "adoção" (43\%) e "implementação" (35\%). Os indicadores menos detalhados (5\%) foram aqueles referentes à dimensão de "manutenção".

\section{Alcance}

De maneira geral, a maioria dos indicadores $(\mathrm{n}=9)$ desta dimensão foi reportada por grande parte dos programas de intervenção (> 50\%). Método de identificação da população-alvo, critérios de inclusão, tamanho da amostra e taxa de participação foram reportados por todos os programas. A descrição, informação demográfica e comportamental da população-alvo e estratégias de recrutamento foram reportados por mais de $70 \%$ dos programas. O custo do recrutamento e utilização de métodos qualitativos para aferir alcance foi mencionado por dois programas.

\section{Efetividade/eficácia}

Nenhum dos componentes desta dimensão obteve $100 \%$ de descrição pelos programas de intervenção. Relato de moderadores, intenção de tratamento e percentual de abandono foram os indicadores mais reportados $(71 \%)$. Na sequência esteve o uso de métodos qualitativos e relato dos resultados primários (57\%), medidas de qualidade de vida (43\%), informações sobre relato de mediadores (29\%), consequências não intencionais (14\%) e custo de efetividade (14\%).

Embora não tenham reportado informações sobre aspectos financeiros, Leme e Philippi ${ }^{25}$ mencionaram como limitação, a falta de avaliação financeira para determinar o custo-efetividade do seu programa de intervenção.

\section{Adoção}

Esta dimensão foi analisada em dois níveis: organizacional e individual. No primeiro nível, $63 \%$ dos respectivos indicadores foram reportados. $\mathrm{O}$ único componente que obteve $100 \%$ de detalhamento foi o número de participantes. Número de elegíveis, convidados e método de identificação do cenário foram informados por $71 \%$ dos estudos. A descrição do local-alvo e do local da intervenção foram os componentes menos informados pelos 
Tabela 1. Indicadores das dimensões do modelo REAIM dos programas de intervenção analisados. Brasil, 2016.

\begin{tabular}{|c|c|}
\hline Alcance & $\%$ \\
\hline Descrição da população-alvo & 71 \\
\hline $\begin{array}{l}\text { Informação demográfica e comportamental } \\
\text { da população-alvo }\end{array}$ & 86 \\
\hline Método de identificação da população-alvo & 100 \\
\hline Estratégias de recrutamento & 86 \\
\hline Critérios de inclusão & 100 \\
\hline Critérios de exclusão & 57 \\
\hline $\begin{array}{l}\text { Número de indivíduos elegíveis e } \\
\text { convidados ao recrutamento }\end{array}$ & 57 \\
\hline Tamanho da amostra & 100 \\
\hline Taxa de participação & 100 \\
\hline Custo do recrutamento & 29 \\
\hline $\begin{array}{l}\text { Utilização de métodos qualitativos para } \\
\text { aferir alcance }\end{array}$ & 29 \\
\hline \multicolumn{2}{|l|}{ Efetividade/Eficácia } \\
\hline Relatório de resultados primários & 57 \\
\hline Relato de Mediatores & 29 \\
\hline Relato de Moderadores & 71 \\
\hline $\begin{array}{l}\text { Intenção de tratamento ou presentes no } \\
\text { acompanhamento }\end{array}$ & 71 \\
\hline Medidas de qualidade de vida & 43 \\
\hline $\begin{array}{l}\text { Aferiu conseqüências não intencionais } \\
\text { (negativos) nos resultados }\end{array}$ & 14 \\
\hline $\begin{array}{l}\text { Porcentagem de abandonos (na conclusão } \\
\text { do programa) }\end{array}$ & 71 \\
\hline Custo-efetividade & 14 \\
\hline $\begin{array}{l}\text { Uso de métodos qualitativos para aferir } \\
\text { eficácia/efetividade }\end{array}$ & 57 \\
\hline
\end{tabular}

\begin{tabular}{lc}
\multicolumn{2}{c}{ Adoção } \\
\hline Nível organizacional \\
Número de elegíveis e convidados (expostos) & 71 \\
Número de participantes & 100 \\
Taxa de participação & 57 \\
Descrição do local-alvo & 43 \\
Critérios de inclusão/exclusão dos cenários & 57 \\
Descrição do local da intervenção & 43 \\
Método de identificação do cenário & 71 \\
Número médio de pessoas servidas pelo & \\
cenário & 57 \\
Nível individual & \\
Elegíveis e convidados (expostos) & 14 \\
Total de participantes que aceitaram & 29 \\
participar & \\
Taxa de participação & 43 \\
Método de identificação dos ajudantes do & 14 \\
programa & \\
Nível de conhecimento dos ajudantes & 29 \\
Critério de inclusão/exclusão para & 14 \\
recrutamento dos ajudantes & \\
Medidas de custo da adoção & 0 \\
\hline & continua
\end{tabular}

Tabela 1. Indicadores das dimensões do modelo REAIM dos programas de intervenção analisados. Brasil, 2016.

\begin{tabular}{|c|c|}
\hline \multicolumn{2}{|l|}{ Implementação } \\
\hline Teorias & 71 \\
\hline Número de contatos da intervenção & 57 \\
\hline Período dos contatos & 29 \\
\hline Duração dos contatos & 57 \\
\hline $\begin{array}{l}\text { Medida em que o protocolo foi executado } \\
\text { conforme o esperado }\end{array}$ & 43 \\
\hline $\begin{array}{l}\text { Consistência na implementação entre } \\
\text { cenários e agentes de execução }\end{array}$ & 14 \\
\hline $\begin{array}{l}\text { Taxas de comparecimento/conclusão dos } \\
\text { participantes }\end{array}$ & 14 \\
\hline Medidas de custo & 14 \\
\hline $\begin{array}{l}\text { Utilização de métodos qualitativos para } \\
\text { medir a implementação }\end{array}$ & 14 \\
\hline \multicolumn{2}{|l|}{ Manutenção } \\
\hline \multicolumn{2}{|l|}{ Nível individual } \\
\hline $\begin{array}{l}\text { Availiação do comportamento individual } \\
\text { após a conclusão da intervenção }\end{array}$ & 43 \\
\hline Abandonos & 0 \\
\hline $\begin{array}{l}\text { Utilização de métodos qualitativos para } \\
\text { aferir a manutenção individual }\end{array}$ & 0 \\
\hline \multicolumn{2}{|l|}{ Nível organizacional } \\
\hline $\begin{array}{l}\text { Relato de alinhamento com a missão do } \\
\text { organização }\end{array}$ & 0 \\
\hline $\begin{array}{l}\text { O programa ainda está em } \\
\text { funcionamento? }\end{array}$ & 0 \\
\hline Se não: razões para a descontinuidade & 0 \\
\hline Se sim: o programa foi modificado? & - \\
\hline O programa foi institucionalizado? & 0 \\
\hline Desgaste & 0 \\
\hline
\end{tabular}

-: Não se aplica

programas (43\%). Quanto ao nível individual da dimensão "adoção", em média, 20\% dos componentes foram mencionados pelos programas. Taxa de participação foi o item mais relatado (43\%). Os componentes sobre medidas de custo não foram informados por nenhum dos estudos.

\section{Implementação}

A utilização de teorias, o número e duração de contatos realizados durante a intervenção foram relatados por $71 \%$ e $57 \%$ dos programas, respectivamente. Aspectos sobre consistência na implementação entre cenários e agentes de execução, taxas de comparecimento e/ou conclusão dos participantes, medidas de custo e utilização de métodos qualitativos foram reportados por apenas $14 \%$ dos programas. 


\section{Manutenção}

Esta dimensão foi analisada em dois níveis. No nível individual, em média, 14\% dos indicadores foram reportados. A avaliação do comportamento individual após a conclusão foi reportada por $43 \%$ dos estudos, ao passo que abandonos e utilização de métodos qualitativos não foram mencionados por nenhuma das publicações. No nível organizacional, nenhum dos respectivos componentes foram informados.

Ribeiro e Alves $^{29}$ explicaram que a falta de avaliação da manutenção do programa foi devida a falta de recursos financeiros.

\section{Resumo dos programas}

Verificou-se que na dimensão "alcance", todos reportaram mais de $50 \%$ da mesma. Na dimensão "efetividade/eficácia", um estudo ${ }^{27}$ não apresentou informações. Os programas "Hábitos Saudáveis, meninas saudáveis" e "Fortaleça sua saúde" reportaram $100 \%$ da dimensão "adoção" - nível organizacional. Quatro programas de intervenção descreveram aspectos sobre "adoção" - nível individual. ${ }^{4,5,19-23,26,28,30,31} \mathrm{Na}$ dimensão “implementação", embora todos tenham apresentado pelo menos um componente, somente os programas "Hábitos saudáveis, meninas saudáveis" e "Fortaleça sua saúde" chegaram a mais de 50\%. Em "manutenção" - nível individual, a maioria dos programas não reportou nenhum item $^{27-30}$. Dentre os que apresentaram informações ${ }^{4,5,19-26,31}$, apenas um componente foi citado (33\%) por cada programa. Em "manutenção" nível organizacional, nenhum componente foi relatado (Tabela 2 ).

\section{Discussão}

O objetivo deste estudo foi revisar sistematicamente os programas de intervenção para AF realizados nas escolas brasileiras com adolescentes de 10 a 19 anos por meio da ferramenta RE-AIM. Nota-se que os programas foram desenvolvidos nos últimos dez anos, englobando escolas públicas, cuja maioria pertencente às regiões Sul e Sudeste do país. A maioria dos programas utilizou estratégias multicomponentes, como educativas e de formação/treinamento de professores, mostrando resultados positivos no que concerne à prática de AF pelos adolescentes. Quanto à análise por meio do modelo RE-AIM, observou-se que a dimensão mais detalhada foi "alcance" e a
Tabela 2. Percentual de detalhamento das dimensões do modelo RE-AIM de acordo com os programas de intervenção para atividade física. Brasil, 2016.

\begin{tabular}{|c|c|c|c|c|c|c|c|}
\hline \multirow{2}{*}{$\begin{array}{c}\text { Programas de } \\
\text { intervenção }\end{array}$} & \multicolumn{7}{|c|}{$\%$} \\
\hline & $\mathbf{R}$ & E & $\mathbf{A}_{0}$ & $A_{i}$ & I & $\mathbf{M}_{i}$ & $\mathbf{M}$ \\
\hline Saúde na Boa ${ }^{4,14-19}$ & 82 & 67 & 75 & 14 & 11 & 33 & 0 \\
\hline $\begin{array}{l}\text { Ribeiro e } \\
\text { Florindo }^{30}\end{array}$ & 64 & 44 & 25 & 43 & 44 & 0 & 0 \\
\hline $\begin{array}{l}\text { Belo Horizonte } \\
\text { Heart Study }{ }^{25}\end{array}$ & 73 & 22 & 25 & 0 & 11 & 0 & 0 \\
\hline $\begin{array}{l}\text { Educa } \\
\text { Física }\end{array}$ & 73 & 56 & 38 & 57 & 33 & 0 & 0 \\
\hline $\begin{array}{l}\text { Hábitos } \\
\text { Saudáveis, } \\
\text { Meninas } \\
\text { Saudáveis }^{20,21}\end{array}$ & 73 & 56 & 100 & 0 & 56 & 33 & 0 \\
\hline $\begin{array}{l}\text { Fortaleça sua } \\
\text { Saúde } e^{5,22}\end{array}$ & 91 & 89 & 100 & 29 & 56 & 33 & 0 \\
\hline TriAtiva $^{23}$ & 64 & 0 & 75 & 0 & 33 & 0 & 0 \\
\hline
\end{tabular}

R: Reach (Alcance); E: Effectiveness/efficacy (efetividade/efiácia); $A_{0}$ : Adoption (Adoção)- Nível organizacional; $A_{i}$ : Adoption (Adoção) - Nível individual; I: Implementation (Implementação); $\mathrm{M}_{\mathrm{i}}$ : Maintanance (Manutenção) - nível individual; $\mathrm{M}$ : Maintenance (Manutenção) - nível organizacional.

menos relatada foi a de "manutenção". De maneira geral, foi possível perceber que os indicadores mais mencionados são aqueles referentes à validade interna do programa.

A homogeneidade em relação à região em que as pesquisas em AF foram realizadas, já vem sendo destacada ${ }^{32}$ e merece atenção para permitir o avanço da área em questão, uma vez que diferentes necessidades são encontradas para cada região. Quanto aos objetivos dos programas, percebe-se que a maior parte trabalhou com foco em mais de um comportamento relacionado à saúde, além da AF (hábitos alimentares ${ }^{4,19-25,31}$, comportamento sedentário ${ }^{5,26,29}$, prevenção da obesidade e excesso de peso ${ }^{24,25,27,30}$ ). Os resultados de Hynynem et al. ${ }^{33}$ sugerem que intervenções com foco em diversos comportamentos são menos efetivas em promover AF. Os autores citam, por exemplo, que AF e comportamento sedentário são comportamentos distintos e, por isso, precisam de intervenções distintas. Esta questão também é abordada por Crutzen et al. ${ }^{34}$, em que se questionou como é que determinada estratégia para um comportamento pode ser, de fato, efetiva para melhorar outro comportamento. Contudo, outros estudos ${ }^{3,14}$, indicaram que evidências são inconclusivas para determinar se intervenções focadas apenas em AF são mais efetivas do que 
quando focadas também em outros comportamentos simultaneamente.

O fato de que a maioria dos programas de intervenção caracterizou-se como multicomponente, ou seja, utilizaram diversos tipos de estratégias, pode ser considerado como aspecto positivo, uma vez que há fortes evidências que programas deste caráter são efetivos para aumentar o nível de AF entre adolescentes, em que pesquisadores recomendaram que política públicas sejam realizadas em escolas com ações multicomponentes ${ }^{3}$.

A dimensão do RE-AIM mais relatada foi "alcance". Detectar o nível do alcance de um programa é passo primordial para direcionar o quanto o programa precisa melhorar para maximizar o número de indivíduos atingidos ${ }^{11}$. Contudo, percebe-se que um item que precisa de mais atenção é a "utilização de métodos qualitativos para aferir alcance", uma vez que estratégias deste caráter como a comunicação com os indivíduos que se deseja atingir, ouvir os relatos e opiniões, pode ser excelente forma de aprimorar o que ainda estiver errado no programa ${ }^{11}$.

Componentes referentes aos custos financeiros foram minimamente relatados em todas as respectivas dimensões, assim como o que acontece com intervenções para AF originárias na América Latina ${ }^{18}$. Essa é uma questão crítica e merece atenção, uma vez que aspectos financeiros podem ser decisivos no processo de tomada de decisão do pesquisador, facilitando (ou impedindo) a transformação dos achados oriundos das pesquisas em realizações de ações concretas para a população ${ }^{18}$. Além disso, a viabilidade financeira determina também, além da implementação e aspectos metodológicos ${ }^{5}$, a sustentabilidade dos programas $^{6}$, cuja descontinuidade dos mesmos está atrelada à interrupção de financiamento. Ribeiro e Alves ${ }^{29}$, por exemplo, relatam que a falta de financiamento impediu a continuidade do programa por mais de um ano. Reis et al. ${ }^{9}$ afirmam que o sucesso de programas nesta área está intimamente interligado com aspectos relacionados a financiamentos para a pesquisa, a fim de permitir que todas as etapas necessárias possam ser realizadas, desde fornecimento de mecanismos à contratação de profissionais. Neste sentido, uma vez que o custo financeiro exerce grande influência na realização de programas de intervenção, informações deste caráter são essenciais para auxiliar gestores e/ou pesquisadores em futuras tomadas de decisões.

$\mathrm{Na}$ dimensão "efetividade/eficácia", um dos itens menos reportados foi, além do custo finan- ceiro supramencionado, o relado de consequências negativas. Relatar tanto os efeitos positivos quanto os negativos permite assegurar que os prejuízos do programa não irão ultrapassar os benefícios ${ }^{10}$. Outro fato que chamou a atenção foi o baixo relato de utilização de mediadores nos programas, visto que os mesmos podem auxiliar na compreensão dos resultados e nos possíveis mecanismos para mudança de comportamento ${ }^{14,35}$.

Assim como verificado em intervenções originárias de outros países ${ }^{36}$, há pouco detalhamento na dimensão "adoção", especificamente no nível individual. O entendimento desta dimensão pode aumentar significativamente a quantidade de pessoas beneficiadas pelo programa ${ }^{11}$. Ter grande "alcance", mas baixa "adoção", por exemplo, tornará o programa restrito em atender a demanda e possível institucionalização ${ }^{11}$. Informações sobre taxa de adoção, características das organizações/ locais que concordam em participar e comparações sobre staffs que concordam ou não em fazer parte do programa são relevantes. Essas questões podem auxiliar futuros pesquisadores a entender melhor quais aspectos limitam a adoção dos programas em diferentes contextos de aplicação $0^{14}$. Detalhamento sobre estes quesitos são fundamentais para avaliar a adaptação, fidedignidade e sustentação do programa ${ }^{14}$, fatos esses que justificam a existência de forte recomendação para que autores detalhem melhor esta dimensão, como forma de aprimorar fatores de validade externa ${ }^{36}$. Esta, por sua vez, poderá contribuir de forma significativa uma vez que demonstra o quanto os achados de um programa são válidos o suficiente para extrapolar para a população em geral ${ }^{37}$.

O pouco esclarecimento na dimensão "implementação" também foi observado em revisão ${ }^{30}$ de programas internacionais. Os autores comentaram sobre a dificuldade em ajustar as muitas informações com os limites de caracteres das revistas como uma possível barreira para tal. Contudo, tais descrições poderão conduzir outras organizações a determinar se irão implementar o programa, auxiliando-os na tomada de decisão $0^{6}$. A informação se o protocolo da pesquisa foi executado conforme o esperado e a consistência na implementação entre cenários e agentes de execução, por exemplo, auxilia a diferenciar qual foi, de fato, o processo seguido na intervenção, em vez de apenas descrever qual seria o cenário ideal ${ }^{36}$. Se a intervenção não foi realizada como originalmente se propunha é justo que o leitor esteja ciente disso para que a avaliação seja com base no que realmente aconteceu e não em cenário imaginário perfeito ${ }^{36}$. Como sugestão, 
Antikainen and Ellis ${ }^{36}$ recomendam a publicação de artigos separados que falem exclusivamente sobre implementação e processo de avaliação.

A dimensão "manutenção" foi a menos relatada. Esse fato foi destacado por Galaviz et al. ${ }^{18}$ em estudos na América Latina, apontando para a limitação de sustentação dos programas. No mesmo sentido, outros estudos obtiveram os mesmos resultados ${ }^{14,38,39}$. McGoey et al. ${ }^{14}$ sugerem que futuras intervenções devam ser executadas de modo que levem em consideração a "manutenção" do programa, pois dessa forma torna-se possível verificar quais aspectos do cenário facilitam ou impedem a institucionalização. Estas questões auxiliam a desenvolver programas de modo que os mesmos sejam mantidos ao longo dos anos, além de auxiliar aos respectivos participantes a continuarem envolvidos ${ }^{11}$. Há extrema necessidade para que a intervenção vá além do campo de pesquisa e seja englobada no sistema, assegurando a "manutenção" e sustentabilidade dos benefícios à saúde 9 .

De modo geral, observa-se tendência de relato para os itens relacionados à validade interna, $\mathrm{o}$ que vem sendo notado em outros estudos ${ }^{14,18,19}$. Antikainen e Ellis ${ }^{36}$ revisaram sistematicamente, por meio da ferramenta RE-AIM, as intervenções baseadas em teorias para AF. Os autores também constataram que, não só intervenções de base escolar para adolescentes, mas também programas comunitários e em diversos outros contextos, envolvendo diferentes populações e faixas etárias também predomina o relato de informações sobre validade interna. Outra similaridade do presente estudo com os programas de intervenção realizados fora do país ${ }^{36}$ é a ênfase dada na comparação de AF pré e pós-intervenção, em detrimento aos relatos sobre protocolos e execução dos programas, bem como sobre aspectos da manutenção. Por outro lado, a falta de descrição sobre as características das populações-alvo verificada por Antikainen e Ellis ${ }^{36}$ opõe-se aos resultados do presente estudo. Os autores hipotetizam que estas informações já estão documentadas nas escolas, o que não acontece normalmente em programas comunitários.

Chama-se a atenção para a necessidade de que pesquisadores reportem aspectos relacionados à validade externa de intervenções (representatividade de staffs e organizações que adotaram a intervenção, se a intervenção foi executada conforme se pretendia, custos e "manutenção") para AF realizadas na América Latina. Para catalisar o processo de pesquisa e de aplicação prática com intuito de permitir o progresso na mudança de comportamento para AF dos jovens é necessário identificar de que forma as intervenções podem ser modificadas e adaptadas para maximizar a participação de toda população ${ }^{14,40}$. Devido a essa falta de informação, torna-se difícil analisar se as intervenções com resultados efetivos podem ser implementadas em outros contextos do mundo real $^{14}$.

Os achados da presente revisão mostram que ainda há muito que avançar em termos de avaliação de programas de AF nas escolas do Brasil. Grande parte dos itens do modelo RE-AIM não são discutidos nos estudos e, ademais, apenas dois trabalhos mencionaram esta carência de informações nas suas limitações ${ }^{25,29}$. Este fato potencializa necessidade apontada pela literatura de que sejam realizados mais esforços para ampliar os resultados de pesquisas para programas de intervenção na população em questão9 .

Aprofundar o conhecimento sobre os mecanismos e processos dos programas de intervenção faz-se necessário para alavancar o número de pessoas e contextos atingidos, alcançando a sustentação das mesmas ${ }^{9}$. Neste sentido, a presente revisão pode ser utilizada como o ponto de partida para o desenvolvimento de futuros estudos sobre programas de intervenção, uma vez que foi identificada elevada carência destas informações no cenário atual do país. Pesquisadores poderão identificar quais dimensões e itens ainda carecem na literatura, e, assim, fornecer mais detalhes sobre os programas. Além disso, os resultados aqui encontrados poderão auxiliar a comunidade científica em como expandir as discussões para além da efetividade dos programas, e incluir em seus debates por quais mecanismos se chegou a tais resultados e que procedimentos foram utilizados para a manutenção dos mesmos.

A presente revisão foi realizada de forma sistemática, com a inclusão de oito bases de dados com temáticas relevantes na área em questão, por meio de critérios rigorosos e bem definidos. Além disso, os estudos foram revisados, em pares, por meio de uma ferramenta validada no Brasil ${ }^{8}$ e com destaque na literatura ${ }^{9}$, o que permite averiguar aspectos tanto de validade interna quanto externa. Por outro lado, cabe destacar que intervenções no Brasil ainda são incipientes, e, possivelmente, programas recentemente publicados na literatura cinzenta, não foram aqui incluídos, como dissertações e teses. 


\section{Conclusão}

Resultados com base na ferramenta RE-AIM enfatizam a necessidade de que programas de intervenção em escolas no Brasil reportem aspectos de validade interna e, principalmente, validade externa para auxiliar em futuras intervenções. Maiores descrições de custos financeiros também são necessárias a fim de esclarecer o custo-benefício dos programas. A partir da avaliação realiza- da por meio do RE-AIM, recomenda-se que pesquisadores realizem esforços para potencializar a "manutenção" de futuras intervenções e contribuir no processo de implementação e ampliação dos programas para AF nas escolas do Brasil. Por fim, com o intuito de determinar se uma intervenção é efetiva e pode ser expandida para condições da vida real, mais informações do modelo RE-AIM precisam ser reportadas para que futuros gestores possam tomar decisões favoráveis.

\section{Colaboradores}

As autoras JA Silva e ECA Gonçalves participaram de todas as etapas de construção do artigo. Os autores DAS Silva e KS Silva auxiliaram na escrita do documento bem como na revisão final do mesmo. 


\section{Referências}

1. Brasil. Health promotion in schools. Rev Saude Publica 2002; 36(4):533-535.

2. Hallal PC. Promoção da atividade física no Brasil: chegou a hora da escola. Rev Bras Atividade Física Saúde 2010; 15(2):76-77.

3. Barbosa Filho VC, Minatto G, Mota J, Silva KS, Campos W, Lopes AS. Promoting physical activity for children and adolescents in low- and middle-income countries: An umbrella systematic review. Prev Med 2016; 88:115-126.

4. Nahas MV, Barros MV, Assis MAA, Hallal PC, Florindo AA, Konrad L. Methods and participant characteristics of a randomized intervention to promote physical activity and healthy eating among Brazilian high school students: the Saude na Boa project. J Phys Act Health 2009; 6(2):153-162.

5. Barbosa Filho VC, Lopes AS, Lima AB, de Souza EA, Gubert FA, Silva KS, Vieira NF, Trompieri Filho N, Araújo TS, Bruin PF, Mota J; "Fortaleça sua Saúde" Working Group. Rationale and methods of a cluster-randomized controlled trial to promote active and healthy lifestyles among Brazilian students: the "Fortaleça sua Saúde" program. BMC Public Health 2015; 15:1212.

6. Benedetti TRB, Schwingel A, Chodzko-Zajko W, Meurer ST, Brito FA, Almeida FA. RE-AIM: uma proposta de avaliação de programas de atividade física. Rev Kairós Gerontol. 2014; 17(2):295-314.

7. Mendonça BC, Oliveira AC, Toscano JJO, Knuth AG, Borges TT, Malta DC, Cruz DK, Hallal PC. Exposure to a Community-Wide Physical Activity Promotion Program and Leisure-Time Physical Activity in Aracaju, Brazil. J Phys Act Health 2010; 7(Supl. 2):S223-S228.

8. Reis RS, Hallal PC, Parra DC, Ribeiro IC, Brownson RC, Pratt M, Hoehner CM, Ramos L. Promoting Physical Activity Through Community-Wide Policies and Planning: Findings from Curitiba, Brazil. J Phys Act Health 2010; 7(Supl. 2):S137-S145.

9. Reis RS, Salvo D, Ogilvie D, Lambert EV, Goenka S, Brownson RC. Scaling up physical activity interventions worldwide: stepping up to larger and smarter approaches to get people moving. Lancet 2016; 388(10051):1337-1348.

10. Glasgow RE, Vogt TM, Boles SM. Evaluating the public health impact of health promotion interventions: the RE-AIM framework. Am J Public Health 1999; 89(9):1322-1327.

11. Almeida FA, Brito FA, Estabrooks PA. Modelo REAIM: tradução e adaptação cultural para o Brasil. Rev Fam Ciclos Vida E Saúde No Contexto Soc 2013; 1(1).

12. Estabrooks P, Dzewaltowski DA, Glasgow RE, Klesges LM. Reporting of validity from school health promotion studies published in 12 leading journals, 19962000. J Sch Health 2003; 73(1):21-28.

13. Binotto $M$, Milistetd $M$, Benedetti T, Almeida F. Programa Universidade Aberta para a Terceira Idade: avaliação do programa por meio do modelo RE-AIM. Rev Bras Atividade Física Saúde 2015; 20(3):309.
14. McGoey T, Root Z, Bruner MW, Law B. Evaluation of physical activity interventions in children via the reach, efficacy/effectiveness, adoption, implementation, and maintenance (RE-AIM) framework: A systematic review of randomized and non-randomized trials. Prev Med 2015; 76:58-67.

15. World Health Organization (WHO). Review of best practice in interventions to promote physical activity in developing countries. Geneva: WHO; 2005.

16. Aires L, Silva G, Martins C, Marques E, Lagoa MJ, Ribeiro JC, Rêgo C, Nascimento H, Pereira PR, Santos-Silva A, Belo L, Mota J. Exercise intervention and cardiovascular risk factors in obese children. Comparison between obese youngsters taking part in a physical activity school-based programme with and without individualised diet counselling: the ACORDA project. Ann Hum Biol 2016; 43(3):183-190.

17. Militao AG, Silva FR, Pereira RMS, Militão ESG, Karnikowski MGO, Campbell CSG. Effects of a recreational physical activity and healthy habits orientation program, using an illustrated diary, on the cardiovascular risk profile of overweight and obese schoolchildren: a pilot study in a public school in Brasilia, Federal District, Brazil. Diabetes Metab Syndr Obes Targets Ther 2013; 6:445-451.

18. Galaviz KI, Harden SM, Smith E, Blackman KC, Berrey LM, Mama SK, Almeida FA, Lee RE, Estabrooks PA. Physical activity promotion in Latin American populations: a systematic review on issues of internal and external validity. Int J Behav Nutr Phys Act 2014; 11(1):77.

19. Hardman CM, Barros MVG de, Lopes AS, Lima RA, Bezerra J, Nahas MV. Efetividade de uma intervenção de base escolar sobre o tempo de tela em estudantes do ensino médio. Rev Bras Cineantropometria Desempenho Hum 2014; 16(0):25.

20. Del Duca GF, Barros MVG, Silva KS, Garcia LMT, Bezerra J, Nahas MV. Intervenção para promoção da atividade física em escolares: efetividade sobre o deslocamento ativo, exercícios de força e estágios de mudança de comportamento. Rev Bras Cineantropometria Desempenho Hum 2014; 16(0):13-15.

21. Silva KS, Barros MVG, Barbosa Filho VC, Garcia LMT, Wanderley Júnior RS, Beck CC, Nahas MV. Implementação da intervenção "Saúde na Boa": avaliação de processo e características dos estudantes permanecentes e não permanecentes. Rev Bras Cineantropometria E Desempenho Hum 2014; 16(0):1.

22. Barbosa Filho VC, Silva KS, Rech CR, Brito ALS, Oliveira ESA, Nahas MV. Mudanças no estilo de vida e na percepção da saúde em estudantes do ensino médio: análise prospectiva do projeto "Saúde na Boa." Rev Bras Cineantropometria E Desempenho Hum 2014; 16(0):55-67.

23. Barros MVG, Nahas MV, Hallal PC, Farias Júnior JC, Florindo AA, Barros SSH. Effectiveness of a schoolbased intervention on physical activity for high school students in Brazil: the Saude na Boa project. J Phys Act Health 2009; 6(2):163-169. 
24. Leme ACB, Lubans DR, Guerra PH, Dewar D, Toassa EC, Philippi ST. Preventing obesity among Brazilian adolescent girls: Six-month outcomes of the Healthy Habits, Healthy Girls-Brazil school-based randomized controlled trial. Prev Med 2016; 86:77-83.

25. Leme ACB, Philippi ST. The Healthy Habits, Healthy Girls; randomized controlled trial for girls: study design, protocol, and baseline results. Cad Saude Publica 2015; 31(7):1381-1394.

26. Barbosa Filho VC, Silva KS, Mota J, Beck C, Silva Lopes A. A Physical Activity Intervention for Brazilian Students from Low Human Development Index Areas: A Cluster-Randomized Controlled Trial. J Phys Act Health 2016; 13(11):1174-1182.

27. Friedrich RR, Caetano LC, Schiffner MD, Wagner MB, Schuch I. Design, randomization and methodology of the TriAtiva Program to reduce obesity in school children in Southern Brazil. BMC Public Health 2015; 15(1):363.

28. Spohr C, Fortes M, Rombaldi A, Hallal P, Azevedo M. Atividade física e saúde na Educação Física escolar: efetividade de um ano do projeto "Educação Física +." Rev Bras Atividade Física Saúde 2014; 19(3):300-313.

29. Ribeiro RQ, Alves L. Comparison of two schoolbased programmes for health behaviour change: the Belo Horizonte Heart Study randomized trial. Public Health Nutr 2014; 17(6):1195-1204.

30. Ribeiro EH, Florindo AA. Efeitos de um programa de intervenção no nível de atividade física de adolescentes de escolas públicas de uma região de baixo nível socioeconômico: descrição dos métodos utilizados. Rev Bras Atividade Física Saúde 2012; 15(1):28-34.

31. Nahas MV, Barros MVG, Goldfine BD, Lopes AS, Hallal PC, Farias Júnior JC, Oliveira ES. Physical activity and eating habits in public high schools from different regions in Brazil: the Saude na Boa project. Rev Bras Epidemiol 2009; 12(2):270-277.

32. Garcia L, Böhm A, Bacil E, Cruz M, Espírito Santo R. A inserção da subárea de Atividade Física e Saúde nos programas de pós-graduação em Educação Física no Brasil. Rev Bras Atividade Física Saúde 2014; 19(2):215-222.

33. Hynynen S-T, van Stralen MM, Sniehotta FF, Araújo-Soares V, Hardeman W, Chinapaw MJ, Vasankari T, Hankonen N. A systematic review of school-based interventions targeting physical activity and sedentary behaviour among older adolescents. Int Rev Sport Exerc Psychol 2016; 9(1):22-44.
34. Crutzen R, de Nooijer J, Brouwer W, Oenema A, Brug J, de Vries NK. Strategies to Facilitate Exposure to Internet-Delivered Health Behavior Change Interventions Aimed at Adolescents or Young Adults: A Systematic Review. Health Educ Behav 2011; 38(1):49-62.

35. Brown H, Hume C, Pearson N, Salmon J. A systematic review of intervention effects on potential mediators of children's physical activity. BMC Public Health 2013; 13(1):165.

36. Antikainen I, Ellis R. A RE-AIM evaluation of theory-based physical activity interventions. J Sport Exerc Psychol 2011; 33(2):198-214.

37. Downs SH, Black N. The feasibility of creating a checklist for the assessment of the methodological quality both of randomised and non-randomised studies of health care interventions. J Epidemiol Community Health 1998; 52(6):377-384.

38. White SM, McAuley E, Estabrooks PA, Courneya KS. Translating physical activity interventions for breast cancer survivors into practice: an evaluation of randomized controlled trials. Ann Behav Med Publ Soc Behav Med 2009; 37(1):10-19.

39. Allen K, Zoellner J, Motley M, Estabrooks PA. Understanding the Internal and External Validity of Health Literacy Interventions: A Systematic Literature Review Using the RE-AIM Framework. J Health Commun 2011; 16(Supl. 3):55-72.

40. Hoehner CM, Ribeiro IC, Parra DC, Reis RS, Azevedo MR, Hino AA, Soares J, Hallal PC, Simões EJ, Brownson RC. Physical Activity Interventions in Latin America: expanding and classifying the evidence. Am J Prev Med 2013; 44(3):e31-e40.

Artigo apresentado em 16/09/2016

Aprovado em 29/01/2018

Versão final apresentada em 31/01/2018 